\title{
émulations
}

\section{Les réponses syndicales au précariat}

\section{Encourager l'employabilité ou la qualification?}

\author{
Benoît Scalvinoni
}

Émulations - Revue de sciences sociales

2019, Varia, En ligne.

\section{Article disponible à l'adresse suivante}

https://ojs.uclouvain.be/index.php/emulations/article/view/scalvinoni

\section{Pour citer cet article}

Benoit Scalvinoni, « Les réponses syndicales au précariat. Encourager l'employabilité ou la qualification? », Émulations, Varia, En ligne. Mise en ligne le 2 mai 2019.

DOI : 10.14428/emulations.varia.024

Distribution électronique : Université catholique de Louvain (Belgique) : ojs.uclouvain.be

(C) Cet article est mis à disposition selon les termes de la Licence Creative Commons Attribution, Pas d'Utilisation Commerciale 4.0 International. http://creativecommons.org/licenses/by-nc/4.0/

Éditeur : Émulations - Revue de sciences sociales / Presses universitaires de Louvain https://ojs.uclouvain.be/index.php/emulations

ISSN électronique : 1784-5734

PUL PRESSES

UNIVERSITAIRES

$\square$ DE LOUVAIN 


\title{
Les réponses syndicales au précariat
}

\author{
Encourager l'employabilité ou la qualification?
}

Benoît Scalvinoni ${ }^{1}$

\begin{abstract}
[Résumé] Si la France est marquée par une précarisation et une dualisation tendancielles de l'emploi, plusieurs projets de réforme ont cherché, à partir des années 2000, à redéfinir l'équilibrage entre la flexibilité de l'emploi et la sécurité des travailleurs. Des organisations syndicales, la CGT et la CFDT, ont également porté des revendications en la matière, soutenant respectivement un nouveau statut du travail salarié et une sécurisation des parcours professionnels. Basée sur une étude de la presse syndicale, cette contribution démontrera que la première intervention syndicale promeut le maintien de la qualification de l'individu dans ou en dehors de l'emploi alors que la seconde soutient son employabilité.
\end{abstract}

Mots clés : emploi, flexicurité, revendications syndicales, chômage, qualification, employabilité.

[Abstract] Because of the precariousness dualisation of employment market, several reform projects have intended, since the 2000s onward, to redefine the balance between employment flexibility and workers' security in France. Two French trade unions, the CGT and the CFDT tried to promote similar claims respectively named "un nouveau statut du travail salarié" and "une sécurisation des parcours professionnels". Studying the trade union press, this article will demonstrate that these two trade union claims have different goals: the first one attempts to maintain individual workers' qualification, while the latter focuses on the employability of people who are currently unemployed.

Keywords: employment, flexicurity, trade unions claims, unemployment, qualification, employability.

\section{Introduction}

Cet article traite de l'acteur du « mouvement syndical » (Bevort, Jobert, 2008), en cherchant à savoir si celui-ci est capable de proposer des visions alternatives au registre dominant caractérisant les actuelles politiques publiques de l'emploi. La précarisation et la dualisation tendancielles de l'emploi remettent aujourd'hui en cause le compromis social instauré pendant les Trente Glorieuses. Le chômage de masse et

\footnotetext{
${ }^{1}$ Sociologue du travail, Université d'Évry-Université Paris Saclay, CPN, France. Cet article est publié à titre posthume, en hommage à son auteur. La rédaction de la revue Émulations adresse ses sincères condoléances aux proches et aux collègues de Benoît Scalvinoni.
} 
l'augmentation du nombre d'emplois précaires mettent à mal le rôle intégrateur des différents modèles d'États-providence (Esping-Andersen, 1990) impuissants à endiguer la profusion des « surnuméraires » ou des « inutiles au monde » (Castel, 1995) - ces individus qui, écartés de l'emploi normal effectué en contrat à durée indéterminée (CDI) à temps plein, économiquement vulnérables et stigmatisés socialement, encourent le risque de se retrouver exclus des systèmes de la protection sociale du fait de carrières professionnelles morcelées et in fine d'intégrer un « précariat » (Paugam, 1997).

En France, au début des années 2000, la recherche de solutions aptes à contrer ces dysfonctionnements du marché du travail a visé un rééquilibrage entre la sécurité des travailleurs et la flexibilité du travail (section 1). En parallèle, des confédérations syndicales ont porté des revendications inédites censées répondre aux aléas de la carrière professionnelle moderne (comme des passages par le chômage ou l'enchainement de contrats courts de travail). Ainsi, la Confédération générale du travail (CGT) revendique la mise en place d'un « nouveau statut du travail salarié (NSTS) » reposant sur une « sécurité sociale professionnelle (SSP) » (section 2), tandis que la Confédération française démocratique du travail (CFDT) promeut pour sa part la mise en œuvre d'une « sécurisation des parcours professionnels (SPP) » (section 3).

Je m'attacherai dans cet article à reconstruire l'élaboration de ces revendications syndicales jusqu'à leur validation par les confédérations syndicales en un axe revendicatif majeur. Pour ce faire, je reconstituerai une historiographie des revendications syndicales basée sur l'analyse des archives de la presse syndicale sur la période 19972008. Les publications étudiées sont La Revue de la CFDT (LR) et Syndicalisme Hebdo (SH) pour la CFDT, Analyses et documents économiques (AE) et Le peuple (LP) pour la CGT. Cette analyse des archives a constitué l'un des terrains empiriques de ma thèse (Scalvinoni, 2013), laquelle a analysé la capacité des syndicats à tenir leur rôle dans les relations professionnelles. Le traitement de cette problématique de recherche a consisté à observer comment ces revendications confédérales ont réussi à influencer et à normaliser les pratiques syndicales repérables à une échelle locale (à savoir l'action des secrétaires généraux des unions régionales et départementales lorraines de la CGT et de la CFDT, et celle de sections syndicales dans l'entreprise). Or, la pleine réalisation des revendications syndicales nécessitait avant tout la conclusion d'accords nationaux interprofessionnels. En l'absence de tels accords, l'analyse de la presse officielle des organisations syndicales a fourni le matériau à partir duquel il m’a été possible de proposer une lecture idéal-typique de ces deux revendications syndicales révélant la stratégie et le positionnement de ces deux syndicats par rapport au discours dominant en matière de traitement du chômage et des transitions dans l'emploi. 


\section{Les principales propositions de lutte contre la précarisation et la dualisation de l'emploi}

Un premier corpus de propositions visant à réguler la précarisation et la dualisation de l'emploi est à mettre à l'actif de juristes et encourage une reconfiguration profonde du droit du travail pour à nouveau encadrer toutes les situations individuelles de travail qu'elles soient précaires ou non, rémunérées ou non. Deux rapports en particulier méritent d'être cités. D'une part, la commission présidée par Jean Boissonnat en 1995², suggère la mise en œuvre d'un « contrat d'activité » devant se substituer à tous les contrats de travail. L'originalité de ce dispositif réside dans son intention d'instaurer une nouvelle norme juridique qui doit conférer à l'individu un statut, des droits, et un revenu, quelle que soit son activité réelle dans le but « d'absorber une partie des multiples dispositifs et des actuels mécanismes de financement de l'insertion, de la formation professionnelle, du chômage ». Mais les contreparties demandées à l'individu quand il bénéficie de cette contractualisation en étant au chômage ou inactif n'étaient que très peu explicitées par la commission Boissonnat. D’autre part, la commission dirigée par Alain Supiot promeut la mise en place d'un « état professionnel de la personne » adossé à « des droits de tirage sociaux ». L'état professionnel de la personne ambitionne le dépassement de «l'engagement contractuel pour intégrer la diversité des formes de travail expérimentées dans la vie humaine » (Supiot, 1999: 86). Il s'agit là aussi de faire en sorte que les périodes d'emploi, de formation, de chômage ou d'inactivité ouvrent toutes droit à une même sécurité statutaire et de ressources « pour assurer la continuité de l'état professionnel des personnes par-delà la diversité des situations de travail et de non-travail » (Supiot, 1999: 86). Dans cette optique, des droits de tirage sociaux sont mis à la disposition du salarié, utilisables librement à la seule condition que ce dernier ait pu préalablement constituer une provision suffisante. Enfin, l'individu en conserverait l'usage malgré une évolution de son statut professionnel.

Un second sous-ensemble de propositions encourage la réappropriation en France des modèles économiques hollandais, autrichien et danois dits de la «Flexicurité ». Ce néologisme provenant de la fusion des termes flexibilité de l'emploi et sécurisation des travailleurs est aujourd'hui très largement incarné par le Danemark qui, dès les années 1980, a procédé à des réformes hybrides alliant le haut niveau de protection sociale associé plutôt aux pays scandinaves et la flexibilisation forte de l'emploi plus typique des pays anglo-saxons. Caractérisé par un faible taux de chômage et une très forte rotation dans l'emploi', le «triangle d'or danois » (Barbier, 2005) repose sur une

\footnotetext{
${ }^{2}$ Boissonnat (1995), Le travail dans Vingt ans, Rapport du groupe présidé par Jean Boissonnat au Commissariat Général du Plan. En ligne, consulté le 5 novembre 2018. URL : http://hussonet.free.fr/trav20-.pdf.

${ }^{3}$ À la période où l'étude est publiée, le taux de chômage officiel est de 6 \% (en dessous de $5 \%$ en 2018), et 700000 danois, soit le tiers de la population active, évoluent chaque année
} 
législation souple en matière de licenciement et de recours aux contrats de travail atypiques, le montant élevé de l'allocation chômage (équivalente à 90 \% du revenu brut d'activité de référence) et une forte activation des chômeurs et de leurs ressources (les allocations chômage sont dégressives et les critères de l'« emploi convenable » que doit rechercher le chômeur de moins en moins restrictifs). La flexicurité danoise s'est depuis imposée comme le modèle économique de référence en Europe (Rey, 2011). Conformément à sa stratégie économique décidée lors du sommet de Lisbonne, l'Union européenne recommande ouvertement aux États membres d'appliquer des réformes inspirées de la flexicurité́, sans toutefois rappeler la nécessité d'allouer des ressources élevées aux chômeurs ou aux personnes amenées à transiter dans l'emploi.

Un troisième bloc de propositions est d'inspiration libérale. Les analyses qui s'y rattachent préconisent une flexibilisation accrue de l'emploi et un assouplissement des règles juridiques en matière d'embauche et de licenciement. Prenons pour exemple le rapport des économistes Pierre Cahuc et Francis Kramarz (2005) qui se réapproprie en le dénaturant le terme cégétiste de «sécurité sociale professionnelle». L’objectif annoncé est de générer une «sécurité d'emploi » tout en réduisant paradoxalement la «protection de l'emploi ». Pour les auteurs de ce rapport, il convient d'instaurer un contrat de travail unique flexible, théoriquement un CDI dont la rupture serait plus aisée ${ }^{5}$, car selon eux, la complexité de la gestion des contrats de travail dissuaderait les employeurs d'embaucher et donc alimenterait le chômage. Les entreprises qui licencieraient seraient déchargées dans un tel système de toutes obligations en matière de reclassement et seraient seulement tenues de verser une taxe à l'État. Cette piste de réforme est à rapprocher d'une proposition antérieure (Blanchard, Tirole, 2003) recommandant d'exonérer l'entreprise de toute responsabilité sociale tout en l'obligeant à verser, dans une logique de "pollueur-payeur », une taxe au service public de l'emploi. Le rapport milite enfin pour l'activation des chômeurs, une dégressivité des allocations chômage et la rétribution des opérateurs du service public de l'emploi en fonction de leurs résultats en matière de reclassement des chômeurs pour non pas " proposer un système indemnisant moins généreusement mais bien un système plus efficace et donc, au final, plus généreux » (Cahuc, Kramarz, 2005 : 45). Un autre exemple que nous pouvons retenir ici, ce sont les propositions présentées par Michel De Virville (De Virville, 2004) aux ministres compétents et plaidants pour une simplification du Code

dans l'emploi. Mais le nombre anormalement élevé de travailleurs handicapés, qui ne sont pas comptabilisés comme des chômeurs, nuance les performances économiques réelles de ce système.

${ }^{4}$ CE (2007), Vers des principes communs de flexicurité : Des emplois plus nombreux et de meilleure qualité en combinant flexibilité et sécurité, Bruxelles, Communication de la Commission européenne Conseil, au parlement européen, au Comité économique et social européen et au Comité des régions.

${ }^{5}$ Une perspective à corréler à la tentative ratée de créer un contrat de première embauche (dérogeant au salaire minimum pour les jeunes) par le gouvernement Villepin en 2006. 
du travail prétextant qu'il serait en l'état inintelligible pour les employeurs, les salariés et les syndicats. Mais sous prétexte de sa simplification, c'est bien une remise en cause des droits des salariés, ce que Laurent Willemez nomme un « détricotage du droit du travail » (Willemez, 2017), qui est recherchée par la généralisation des contrats à durée déterminée «d'usage » ou l'instauration d'un « contrat de projet». De tels contrats de travail seraient en effet plus faciles à rompre par le biais de licenciements "négociés » ou " volontaires », et vont complètement dans le sens des propositions en la matière des organisations patronales ${ }^{6}$.

Tendanciellement, les projets de réforme présentés ci-dessus préconisant un rééquilibrage entre la sécurité des travailleurs et la flexibilité de l'emploi recommandent essentiellement une flexibilisation maximale de l'emploi. En contrepartie, la sécurité des travailleurs se réduit pour les salariés multipliant les emplois précaires, les inactifs et les chômeurs, au bénéfice de ressources minimales, mais souvent faibles, dédiés au retour à l'emploi. Dans ce contexte, les organisations syndicales postulant qu'un même type de rééquilibrage est nécessaire arrivent-elles à revendiquer des dispositifs et des mesures véritablement alternatifs?

\section{Le nouveau statut du travail salarié et la sécurité sociale professionnelle à la CGT}

À la CGT, l'origine du « nouveau statut du travail salarié », NSTS, est à corréler au projet défendu par la centrale syndicale d'obtenir des « avancées sociales solidaires » et de parvenir à un "plein emploi solidaire $»^{7}$, notamment dans le cadre des négociations interprofessionnelles encadrant la réforme de la formation professionnelle continue confiée, le 30 mars 1998, à Nicole Perry, Secrétaire d'État à la formation professionnelle. Pour la CGT, l'enjeu décisif est alors la reconnaissance des qualifications individuelles pour garantir le «droit pour chacun à un réel déroulement de carrière » ${ }^{8}$ qui, en termes concrets, pourrait prendre la forme d'un droit individuel à la formation qualifiante reconnu par des garanties collectives et correspondant à $10 \%$ du temps de travail.

En 1999, à l'issue du 46e congrès confédéral de Strasbourg, la CGT revendique des " garanties collectives modernes » dont les droits au travail (à un emploi stable à temps plein), à l'égalité des sexes dans l'emploi, à un développement de carrière, un droit effectif à la formation, des droits sociaux et, enfin, des libertés syndicales. On retrouve là

\footnotetext{
${ }^{6}$ MEDEF (2004), Moderniser le droit du travail les 44 propositions du MEDEF, Direction des relations sociales.

${ }^{7}$ Source : $L P, \mathrm{n}^{\circ} 1469$.

${ }^{8} L P, \mathrm{n}^{\circ} 1482-83$.
} 
l'ossature embryonnaire du futur NSTS, même si ce terme précis n'est pas encore usité9. Successeur de Louis Viannet à la tête du syndicat, Bernard Thibaut déclare qu'il convient d'évoluer d'une «protection passive à une sécurité active » dans l'emploi ${ }^{10}$ et que les garanties et la qualification des individus deviennent «transversables et transférables $»^{11}$.

En marge de l'élaboration du nouveau statut du travail salarié, la SSP consistera à « établir une sécurité sociale d'emploi et de formation tout au long de la vie professionnelle qui garantisse une continuité du salaire, le droit à la formation qualifiante, à la validation des acquis professionnels et au reclassement $»^{12}$. En décembre 2001, un socle de sept droits salariaux forme le prototype réactualisé du NSTS dont un « droit à l'emploi » stipulant que le CDI à temps plein doit devenir la règle d'embauche, un « droit à la formation tout au long de la vie » afin que tout salarié dispose de $10 \%$ du temps de travail pour la formation, soit 3,75 ans pour une carrière de 37,5 annuités, un « droit à la reconnaissance, au déroulement de carrière, au pouvoir d'achat » posant par principe que tout salarié devrait doubler son salaire d'embauche à la fin de sa carrière ${ }^{13}$. L’année 2002 voit la multiplication des références explicites au NSTS qui acquiert donc sa terminologie définitive. En revanche, la SSP demeure objectivement un mystère pour les lecteurs de la presse du syndicat qui la présentent, dans le meilleur des cas, comme une " garantie nationale interprofessionnelle permettant à chaque individu de disposer du droit à la pérennité de son contrat de travail, même si l'entreprise ferme, se restructure ou délocalise toutes ou une partie de ses activités $»^{14}$. Le NSTS et la SSP sont cependant à l'ordre du jour du $47^{\mathrm{e}}$ congrès confédéral de Montpellier de 2003, quoique la confédération concède à son issue que, pour certains de ses militants «c'est un truc fourre-tout dont le contenu reste vague $»^{15}$.

Le 10 juin 2005, Maryse Dumas, élue au Bureau confédéral et personnalité de la CGT parmi les plus impliquées dans la promotion de la revendication confédérale définit la SSP comme « une nouvelle garantie interprofessionnelle assurant chaque salarié d'un droit véritable à l'emploi, [...] une sécurité sociale professionnelle qui respecte son droit à la progression de sa carrière et de son salaire, à la pérennité de son contrat de travail

\footnotetext{
${ }^{9}$ La CGT se réfère tour-à-tour à « une meilleure sécurité d'emploi » $\left(L P, n^{\circ} 1486\right)$, une « sécurité renouvelée » dans l'emploi $\left(A E, \mathrm{n}^{\circ} 78\right)$, de « nouvelles règles permettant une sécurité salariale » (LP numéro spécial, 1496-99), ou encore à un «nouveau statut du salarié » (LP, $\left.\mathrm{n}^{\circ} 1541\right)$.

${ }^{10} L P, n^{\circ} 1518$.

${ }^{11} A E, \mathrm{n}^{\circ} 79$.

${ }^{12} A E, \mathrm{n}^{\circ} 87-88$.

${ }^{13} L P, n^{\circ} 1551$.

${ }^{14} L P, n^{\circ} 1558$.

${ }^{15} L P, \mathrm{n}^{\circ} 1573$.
} 
même si l'entreprise ferme ou supprime l'emploi $»^{16}$. Et pour que les militants puissent se saisir eux-mêmes de la revendication confédérale, des comparaisons à des dispositifs existants sont rapportées dans les journaux du syndicat. Sont citées une énième restructuration du site Areva de Saint-Ouen qui a mis en place un «dispositif exceptionnel d'une durée de 36 mois [...] avec maintien du salaire», ou l'instauration, le 14 avril 2006, du contrat de transition professionnelle ${ }^{17}$ (CTP) expérimenté dans sept bassins d'emplois même s'il organisait « une contractualisation du chômage » et non « une pérennisation du contrat de travail $»^{18}$. La conclusion d'un accord collectif au sein de l'enseigne « La Samaritaine » serait enfin un exemple de « bonne pratique » de SSP à l'échelle de l'entreprise dans la mesure où le salarié licencié dans le cadre d'un plan de sauvegarde de l'emploi « qui n'obtiendrait pas d'offres valables de reclassement continuerait à percevoir son salaire, sans date limite $»^{19}$.

Le NSTS et la SSP sont à nouveau débattus à l'occasion du $48^{e}$ congrès confédéral de la CGT se déroulant à Lille en avril 2006. Si la revendication ne représente que quelques pages du compte-rendu officiel qui en compte près de 200, Bernard Thibaut réaffirme que la revendication « brevetée CGT » consiste à garantir une progression de la carrière malgré les aléas de la vie professionnelle ${ }^{20}$. Mais il est rapporté plus loin dans le même document qu'il demeure « une hésitation, dans les syndicats, à prendre cette question à l'offensive ». Il est vrai que 160 des 993 amendements au document d'orientation préparatoire au congrès visaient « à effacer du document la référence au nouveau statut du travail salarié et à la sécurité professionnelle ». À l'heure actuelle encore, la SSP ne fait pas l'unanimité au sein des militants de la CGT, en raison qu'elle abandonne de facto la position de principe d'interdiction stricte des licenciements (Cingolani, Friot, 2007). Malgré cette contestation interne, le NSTS et la SSP constituent une motion soumise au vote lors du congrès confédéral et au terme duquel ils deviennent un axe revendicatif officiel de la confédération syndicale.

La commission exécutive confédérale de la CGT organise un forum national dédié au NSTS réunissant 250 participants en février 2007. Dans la foulée, une brochure de 24 pages tirée à 50000 exemplaires intitulée « conquérir un nouveau statut du travail salarié » présente la revendication confédérale dans sa version définitive. Huit droits

\footnotetext{
${ }^{16} L P, \mathrm{n}^{\circ} 1615$.

${ }^{17}$ Rappelons que le CTP était proposé aux salariés licenciés dans le cadre de licenciements économiques. Bénéficiant au maximum 12 mois d'une allocation de transition professionnelle équivalente à $80 \%$ de leur précédent salaire, les bénéficiaires du CTP étaient en revanche fortement contraints (dans le cadre d'un accompagnement individualisé renforcé) à rechercher activement un emploi.

${ }^{18} L P, \mathrm{n}^{\circ} 1623$

${ }^{19} L P, \mathrm{n}^{\circ} 1626$

${ }^{20} L P$, supplément au $n^{\circ} 1632$.
} 
attachés à l'individu, dont celui à une SSP qui consiste à « garantir aux salariés dont l'emploi est, pour diverses raisons, supprimé, l'intégralité de leurs droits et singulièrement la poursuite de leur contrat de travail jusqu'à l'obtention d'un nouveau contrat de travail $»^{21}$. Est également précisé que le salarié pris en charge dans le cadre de la SSP bénéficierait d'une allocation équivalente à 90 \% de son précédent salaire. Des « repères revendicatifs $\aleph^{22}$, des fiches pratiques synthétiques ${ }^{23}$, mises en lignes depuis sur le portail Internet de l'organisation complètent les efforts de promotion de la revendication syndicale.

On peut enfin signaler que la CGT s'est servie des négociations nationales interprofessionnelles sur la modernisation du marché du travail ouvertes le 7 septembre 2007 pour promouvoir et tenter de mettre en œuvre les principes du NSTS. En positif, l'accord collectif conclu le 11 janvier 2008 réalise à nouveau la transférabilité de certains droits (le MEDEF parle de leur "portabilité »). En négatif, il «accroît la flexibilité des

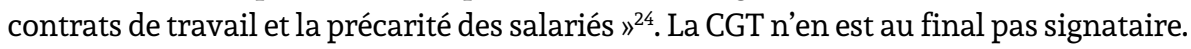
Une décision ratifiée en interne par 90 unions départementales et 23 fédérations professionnelles (soit une abstention). À ce niveau des relations professionnelles, seule la mise en place du droit individuel à la Formation par un accord national interprofessionnel conclu en 2003 demeure véritablement, pour la CGT, « une première pierre de la construction d'un socle interprofessionnel de droits appartenant à la personne du salarié, transférables et cumulables, d'une entreprise à l'autre, [...] en perspective d'un nouveau statut du travail salarié $»^{25}$.

\section{La sécurisation des parcours professionnels à la CFDT}

C'est pour inverser le phénomène de la désyndicalisation (Andolffato, Labbé, 2009) que la CFDT compte développer dès 1998 un « syndicalisme d'adhérents » atteignable grâce à un « véritable projet collectif » incluant une « transformation sociale »" ${ }^{26}$. Il s'agit plus précisément pour la centrale syndicale de reconstruire un compromis rééquilibrant les

\footnotetext{
${ }^{21} L P, n^{\circ} 1645$.

22 Six des 35 repères revendicatifs publiés traitent directement ou indirectement du NSTS et/ou de la SSP.

${ }^{23} L P$, supplément au $n^{\circ} 1649$.

${ }^{24}$ CGT (2008), Négociation sur le marché du travail : la CGT ne signe pas l'accord, communication confédérale. En ligne, consulté le 5 novembre 2018. URL : https://cgt.fr/Negociation-sur-lemarche-du.html.

${ }^{25} L P, n^{\circ} 1583$.

${ }^{26} L R, \mathrm{n}^{\circ} 7$.
} 
rapports entre l'économique et le social et étendu à toutes les strates du salariat ${ }^{27}$. La problématique posée explicitement par le syndicat est la suivante : « comment assurer une continuité des droits sociaux au-delà de la pluralité des situations de travail » alors que les « garanties collectives d'hier n'assurent plus forcément contre les risques d'aujourd'hui $»^{28}$ ?

En 2002, le congrès confédéral de Nantes voit la succession de François Chérèque à la place de Nicole Notat au poste de Secrétaire général du syndicat. Le rapport d'activité (adopté à la plus large majorité dans toute l'histoire de la CFDT) entérine l'objectif d'aboutir à un «plein emploi de qualité ». Le développement de l'épargne salariale, la généralisation de la réduction du temps de travail, la garantie du droit au reclassement pour tous les salariés licenciés, ou le développement de la formation en alternance comptent alors parmi les missions subordonnées à cet objectif. Ces différents principes composeront plus tard le fil conducteur de la SPP. On le voit, la SPP ne marquera pas une rupture stratégique pour la CFDT. Au contraire, elle nommera et normalisera des pratiques entretenues de longue date par le syndicat, mais fédérées autour d'une même bannière sémantique.

C'est en 2003 que l'on repère la première référence explicite à la SPP dans les archives étudiées. Selon la CFDT, elle doit allier « flexibilité, mobilité et surtout sécurité " $^{29}$. Dans le domaine de l'emploi, et pour lutter contre la précarisation et l'instabilité de certaines catégories de salariés, la CFDT veut, dans une logique assumée de discrimination positive, focaliser son intervention sur les individus « les plus éloignés du marché du travail ». En cas de licenciement, tous les appareillages disponibles doivent servir un objectif de réinsertion dans l'emploi: «cellules de reclassement, proposition d'embauches, formation, reconversion, aide à la mobilité ». Ainsi définie, la mise en œuvre du plan d'aide au retour à l'emploi (PARE) issu des négociations nationales interprofessionnelles de 2001 sur l'assurance-chômage insère rétroactivement le champ de la SPP. Conjointement, les dispositifs en matière de formation professionnelle doivent être au « au service d'une stratégie d'emploi centrée sur les transitions professionnelles », dotant l’individu de " compétences » garantissant « le retour à l'emploi ». En développant la validation des acquis de l'expérience (VAE) et en mettant en place un passeport formation, la CFDT entend « faire émerger chez tous les individus l'envie de se former et la possibilité de construire des projets de qualification ».

En prévision de la tenue en juin 2006 à Grenoble du 46e congrès confédéral, François Chérèque rappelle le principe matriciel de la SPP qui consiste à obtenir « de nouvelles garanties à la flexibilité [...] pour la rendre acceptable $»^{30}$. De la lecture du projet de

\footnotetext{
${ }^{27} L R, n^{\circ} 11$.

${ }^{28} \angle R, \mathrm{n}^{\circ} 30$.

${ }^{29} L R, \mathrm{n}^{\circ} 58$.

${ }^{30} L R, \mathrm{n}^{\circ} 73$.
} 
résolution préparatoire au congrès modifié sur la base des 2224 amendements déposés en préalable au Bureau confédéral, on retiendra que la SPP constitue l'un des cinq axes prioritaires de « la stratégie syndicale offensive " $^{31}$ voulue par la CFDT. Pour « sécuriser la mobilité dans l'emploi », la SPP promeut le développement de dispositifs permettant la transférabilité des droits (notamment en matière de santé), le renforcement de l'accompagnement individualisé des chômeurs, l'élargissement de la durée et de la couverture de la convention de reclassement personnalisée (CRP), la meilleure accessibilité à la VAE.

Ultérieurement, Gaby Bonnand, secrétaire national à la CFDT, déclare que «l'aide aux chômeurs sous forme de soutien à la recherche d'emploi devient la priorité de la CFDT. Cette dernière a été à l'initiative du passage progressif d'un système d'indemnisation passif à la mise en place d'un accompagnement de l'indemnisation, de mesures d'aide au retour à l'emploi » ${ }^{32}$. À l'instar de la flexicurité danoise, la SPP consiste à doter l'individu de ressources et de droits adéquats en contrepartie de son engagement à entamer une démarche active de retour ou de transition dans l'emploi. En ce sens, les conventions de conversion, l'accompagnement individualisé des chômeurs, les conventions de coopération, le PARE et le projet d'action personnalisé sont autant de dispositifs qui illustrent ou matérialisent la SPP.

L'historiographie de la revendication syndicale se confond ensuite totalement avec les négociations nationales interprofessionnelles sur la modernisation du marché du travail, à l'image de la déclaration suivante de François Chérèque datée du 27 septembre 2007:

La sécurisation des parcours professionnels est pour nous le fil rouge de ce vaste chantier. [...] Cela signifie de [sic] faire en sorte que les ruptures dans la carrière soient des moments de rebond et d'évolution professionnelle. [...] Nous proposons, pendant les périodes de rupture, un accompagnement fort par le service public de l'emploi. Cette période pourrait ainsi être mise à profit par le demandeur d'emploi pour se former, valider ses acquis, se réorienter ${ }^{33}$.

On rappellera que ces négociations interprofessionnelles débutent en octobre 2006 à l'initiative de l'ensemble des partenaires sociaux à l'exception notable de la CGT, et que ceux-ci s'accordent à rénover l'assurance chômage et le contrat de travail ainsi qu'à négocier la sécurisation des parcours professionnels. Elles s'achèvent le 11 janvier 2008, par la validation d'un accord national interprofessionnel. La CFDT se félicite que ce dernier instaure: «la création d'un cadre collectif pour encadrer les ruptures individuelles du contrat de travail, avec notamment un niveau d'indemnisation

${ }^{31} \mathrm{SH}, \mathrm{n}^{\circ} 3073$, supplément.

${ }^{32} \angle R, n^{\circ} 79$.

${ }^{33} \mathrm{SH}, \mathrm{n}^{\circ} 3128$. 
garanti ; la mise en place d'un fonds spécifique pour la formation professionnelle qualifiante des personnes les plus éloignées de l'emploi ; ou encore une nouvelle mécanique de mutualisation de certains droits, premier pas vers leur transférabilité $\$^{34}$. François Chérèque conclut à propos de la conclusion de cet accord : « il n'y a pas d'espace dans lequel le patronat est parvenu à accroître la précarité sans contrepartie. [...] À chaque fois que la flexibilité augmente, de nouvelles garanties sont apportées aux salariés $»^{35}$.

Forte de cet accord cohérent avec sa stratégie de transformation sociale par le biais du réformisme, l'agenda des relations professionnelles, et en particulier les négociations ultérieures sur la formation professionnelle, la gestion prévisionnelle des emplois et des compétences et l'assurance-chômage, sont autant de fenêtres de tir dont disposent la CFDT pour poursuivre la matérialisation de la SPP par des accords collectifs. Systématiquement, l'objectif visé sera « d'assurer à chaque salarié les garanties indispensables pour un parcours professionnel assorti d'un accroissement des compétences, de l'amélioration de son employabilité lorsqu'il est en activité dans une entreprise $\aleph^{36}$. Au final, ces trois chantiers de négociation donnent lieu à la conclusion d'accords nationaux interprofessionnels que la CFDT, malgré des « regrets », des « hésitations » assimile systématiquement à la continuation de la sécurisation des parcours professionnels.

\section{Conclusion}

Quels enseignements tirer de cette analyse synthétique de ces deux revendications syndicales ? Si les revendications du NSTS et de la SSP à la CGT et de la SPP à la CFDT entendent l'une et l'autre à lutter contre les effets de la dualisation et de la précarisation de l'emploi, les perspectives soutenues sont divergentes. Le NSTS cherche à attacher à la personne un socle de droits (relatifs au salaire, à la retraite, à la formation, etc.) qui ne seraient donc plus liés à l'emploi pour, dans une perspective assez inédite, les déconnecter totalement de l'exercice concret du travail. Des droits garantis à un niveau interprofessionnel, transférables et cumulables en cas de changement d'employeur ou d'inactivité. Au titre de ces garanties nouvelles, la SSP consisterait plus spécifiquement s'agissant d'un licenciement à maintenir le contrat de travail, la rémunération et les droits acquis jusqu'alors par l'individu. La revendication portée par la CGT participe in fine au maintien, et par extension au développement par un effet de cliquet de la « qualification salariale » de l'individu (Charlier et al., 2003), c'est-à-dire au critère politique un peu abstrait par lequel il est possible de déterminer le niveau de rémunération ou encore la forme statutaire de l'emploi. De son côté, la SPP repose sur un meilleur

\footnotetext{
${ }^{34} \mathrm{SH}, \mathrm{n}^{\circ} 3158$.

${ }^{35} \mathrm{SH}, \mathrm{n}^{\circ} 3153$.

${ }^{36} \mathrm{SH}, \mathrm{n}^{\circ} 3163$.
} 
accompagnement des individus dans leurs mobilités professionnelles avec un soutien renforcé au moment du chômage. La CFDT milite dans cette perspective pour le perfectionnement de la VAE, le développement de droits individuels, l'amélioration de l'indemnisation du chômage et de la gamme des outils d'aide au retour à l'emploi qui, dans une logique de discrimination positive, se destinent aux individus les plus éloignés de l'emploi. En ce sens, la revendication de la CFDT encourage davantage la réduction de «l'inemployabilité » des individus (Ebersold, 2001), cette définition assez négative de l'individu qui mesure sa distance à l'emploi en fonction principalement de ses manques (en termes de qualification, de compétences, d'expérience professionnelle, etc.).

Différentes dans leurs objectifs, les revendications syndicales se positionnent également différemment par rapport au registre actuellement dominant des politiques publiques de l'emploi. Les réformes appliquées par les derniers gouvernements successifs et les accords nationaux interprofessionnels généralement validés par les partenaires sociaux convergent en un mouvement général qui contribue à insérer le chômeur ou le salarié instable dans un parcours individualisé de réinsertion ou d'évolution dans l'emploi, et favorise un principe général dédié au maintien ou à la régénération de son employabilité, une orientation idéologiquement plus proche de la SPP soutenue par la CFDT. Concrètement, rares sont les dispositions mises en œuvre au niveau national qui véhiculent un principe idéologique intrinsèque de maintien de la qualification salariale, conformément au NSTS et à la SSP promus par la CGT. En définitive, le droit individuel à la formation aura été, au niveau interprofessionnel, la seule mise en pratique de la revendication confédérale soutenue par la CGT. Mais dans le cadre de la réforme de la formation professionnelle entamée en 2014, le compte personnel de formation l'a depuis remplacé. Impliquant un entretien professionnel et un conseil en évolution professionnelle, le compte personnel de formation tend manifestement à améliorer l'employabilité de l'individu. De son côté, la CFDT peut au contraire multiplier les exemples concrets et labellisés de la SPP. Une illustration récente est la mise en place en $2011 \mathrm{du}$ contrat de sécurisation professionnelle qui s'est substitué au CTP et à la CRP et qui renforce l'activation du chômeur qui en bénéficie.

Par le biais de sa revendication syndicale de SPP et sa propension à être le syndicat signataire du plus grand nombre d'accords collectifs, la CFDT continue donc à se positionner comme le partenaire privilégié du patronat et des pouvoirs publics dans le système des relations professionnelles, tandis que la CGT tend plutôt à rester l'acteur le plus contestataire. Mais indépendamment des stratégies singulières adoptées par les deux confédérations syndicales, force est de constater que la légitimité et la capacité d'action de l'acteur syndical tendent de plus en plus à être remises en cause et effritées. La récente réforme des règles de la représentativité syndicale au terme de laquelle se sont malgré tout maintenues les cinq confédérations syndicales qui bénéficiaient d'une présomption irréfragable de représentativité depuis 1966, la loi El Khomri d'août 2016 relative au travail, à la modernisation du dialogue social et à la sécurisation des parcours professionnels, et les ordonnances Pénicaud adoptées par l'Assemblée nationale 
en novembre 2017 sont autant d'offensives visant à contourner l'acteur syndical déjà affaibli par une désyndicalisation et une division plus fortes que jamais. Dans ce contexte, les organisations syndicales se retrouvent « prises en tenaille (Glaymann, 2007), acculées à la défensive et contraintes dans les négociations collectives à se contenter de faire le choix du moindre mal. La réforme à venir de l'assurance-chômage ne risqueelle pas de confirmer cette tendance ? Si l'indemnisation du chômage doit s'étendre, sous conditions, aux salariés démissionnaires ou indépendants, le contrôle plus systématique des chômeurs et de leur démarche active de retour à l'emploi, la hausse des sanctions voire la mise en place de la dégressivité des allocations chômage sont tout autant des pistes de réflexion envisagées qui sont pour le moins éloignées de la SSP et davantage encore du NSTS.

\section{Bibliographie}

Andolfatto D., LabBe D. (2009), Toujours moins! Déclin du syndicalisme à la française, Paris, Gallimard (« Le débat »).

BARBIER J.-C. (2005), « Apprendre vraiment du Danemark : réflexion sur le miracle danois », Esprit, $n^{\circ} 316$, p. 54-64.

BeVort A., JOBERT A. (2008), Sociologie du travail : les relations professionnelles, Paris, Armand Colin.

Blanchard O., Tirole J. (2003), Protection de l'emploi et procédures de licenciement, Paris, La Documentation française (« Les rapports du Conseil d'analyse économique »).

Cahuc P., Kramarz P. (2005), De la précarité à la mobilité : vers une sécurité sociale professionnelle, Paris, La documentation française (« Collection des rapports officiels »).

CASTEL R. (1995), Les métamorphoses de la question sociale. Une chronique du salariat, Paris, Fayard (« Espace du politique »).

Charlier M., Colin T., Grasser B., RyK G. (2003), La construction sociale des frontières entre la qualification et la non qualification, Rapport de recherche pour la DARES.

Cingolani P., FriOt B. (2007), Syndicalisme et action collective face aux différentes formes de flexibilité de l'emploi et du travail, Rapport de recherche pour la DARES.

EBERSOLD S. (2001), La naissance de l'inemployable ou l'insertion aux risques d'exclusion, Rennes, Presses Universitaires de Rennes (« Le sens social »).

ESPING-ANDERSEN G. (1990), The Three Worlds of Welfare Capitalism, Cambridge, Princeton University Press.

Glaymman D. (2007), L’intérim, Paris, La Découverte (« Repères »). 
PAugam S. (1997 [1991]), La disqualification sociale. Essai sur la nouvelle pauvreté, Paris, Presses universitaires de France («Sociologies »).

REY F. (2011), « Les usages du concept de modèle dans la construction du modèle social danois », Revue française de socio-économie, vol. 1, n 7, p. 169-189.

SCALVINONI B. (2013), Une analyse de l'expérimentation et de diffusion en Lorraine des revendications syndicales de "la prise en charge des discontinuités d'emploi » portées au niveau confédéral par la CGT et par la CFDT, thèse de doctorat, Nancy, Université de Lorraine.

Supiot A. (dir.) (1999), Au-delà de l'emploi : transformations du travail et devenir du droit du travail en Europe, Paris, Flammarion (« Divers Sciences »).

Virville (DE) M. (2004), Pour un code du travail plus efficace, Paris, La Documentation française (« rapports officiels »).

WiLLEMEZ L. (2017), Le travail dans son droit, Issy-les-Moulineaux, LGDJ (« Contextes »). 